${ }^{(1)}$ Residente de Primer año de Geriatría, Pontificia Universidad Javeriana, Hospital Universitario San Ignacio. Bogotá, Colombia.

${ }^{(2)}$ Médico Geriatra, Hospital Universitario San Ignacio, Pontificia Universidad Javeriana. Bogotá, Colombia.

Correspondencia: Rodrigo Heredia, correo electrónico: herediar@javeriana.edu.co Recibido: 10/06/15. Aceptado: 20/06/15.

\title{
Sarcopenia en enfermedad pulmonar obstructiva crónica: rehabilitación pulmonar como factor fundamental en el tratamiento multidimensional de los adultos mayores
}

\section{Sarcopenia in COPD: pulmonary rehabilitation as a fundamental factor in multidimensional treatment}

Miguel GeRmÁn Borda, MD.(1); Rodrigo HeRedia, MD. ${ }^{(2)}$

\section{Referencia}

Jones SE, Maddocks M, Kon SS, et al. Sarcopenia in COPD: prevalence, clinical correlates and response to pulmonary rehabilitation. Thorax. 2015;70:213-8.

\section{Pregunta}

¿Cuál es la prevalencia y el impacto de la sarcopenia en la enfermedad pulmonar obstructiva crónica (EPOC) y el efecto de la rehabilitación pulmonar?

\section{Métodos}

\section{Diseño}

La respuesta a la rehabilitación pulmonar se evaluó con un estudio observacional prospectivo

\section{Sujetos}

Se aplicaron los criterios del International European Working Group on Sarcopenia in Older People (EWGSOP) en 622 pacientes con EPOC estable (figura 1). De este grupo, 43 recibieron rehabilitación pulmonar.
La medición de la fuerza de prensión se estimó con un dinamómetro, siendo el valor normal para hombres de $\geq 30 \mathrm{~kg}$ y para mujeres de $\geq 20$ $\mathrm{kg}$. La medición de la masa muscular se estimó mediante bioimpedancia e índice de masa muscular esquelético.

\section{Cegamiento}

No aplica.

\section{Periodo}

Se estudiaron pacientes captados entre abril de 2011 y enero de 2014.

\section{Lugar}

Pacientes ambulatorios de la consulta externa de Neumología del Harefield Hospital, Middlesex, Reino Unido.

\section{Población}

Pacientes de la consulta externa de Neumología con diagnóstico de EPOC.

\section{Intervención}

Rehabilitación pulmonar: programa ambulatorio multidisciplinar 


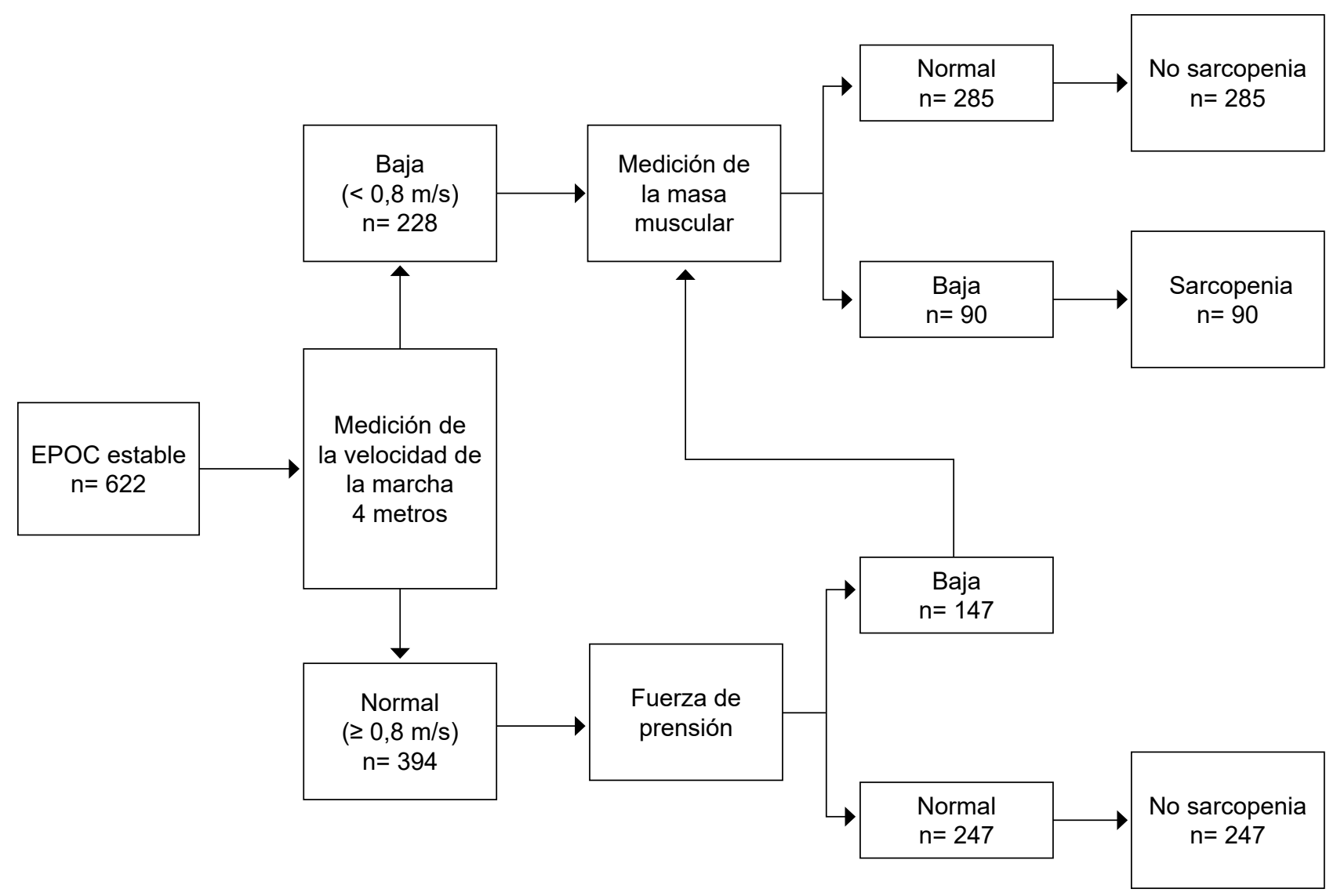

Figura 1. Flujograma para el diagnóstico de sarcopenia según los criterios de la EWGSOP.

de ejercicio y de educación de ocho semanas que comprendía dos sesiones supervisadas y al menos una adicional en casa por semana. Las sesiones supervisadas incluían una hora de ejercicio y una hora de educación. El ejercicio físico fue individualizado, y de acuerdo con la práctica del Reino Unido, principalmente de naturaleza aeróbica. Las sesiones de educación cubrieron una variedad de temas de autocuidado, incluyendo ejercicio, uso de medicamentos, dieta, estrategias para enfrentar la enfermedad, aumento de la actividad física y reconocimiento y manejo de las infecciones.

La respuesta a la rehabilitación pulmonar se calculó en pacientes con sarcopenia que habían asistido al menos al $50 \%$ de las sesiones supervisadas ( $\geq 8 / 16$ ) y habían completado una evaluación posrehabilitación pulmonar, los cuales fueron comparados con sujetos que no presentaron sarcopenia.

\section{Resultados}

La prevalencia global de la sarcopenia fue de 14,5\% (IC95\% 11,8\% a 17,4\%). Los pacientes con sarcopenia fueron significativamente mayores, tenían menor $\mathrm{VEF}_{1}$, fuerza del cuádriceps reducida, menor capacidad de ejercicio, menor desempeño funcional, menor actividad física y peor estado de salud en comparación con aquellos sin sarcopenia (tabla 1).

Los pacientes con y sin sarcopenia asistieron a un promedio de 13 (DS 2) y 13 (DS 3) sesiones de rehabilitación pulmonar, respectivamente. Después de la rehabilitación, no hubo diferencias significativas respecto a los desenlaces de la composición corporal, el rendimiento funcional o el estado de salud entre los pacientes con y sin sarcopenia.

Aún más importante fue que los pacientes con sarcopenia después de la rehabilitación, mejoraron 
Tabla 1. Características basales y prevalencias en sujetos con enfermedad pulmonar obstructiva crónica.

\begin{tabular}{|lrcc|}
\hline & Sin sarcopenia & \multicolumn{1}{c|}{ Sarcopenia } & \multicolumn{1}{c|}{ Valor de $\mathbf{p}$} \\
$\mathrm{n}$ & 220 & 90 & \\
Edad & $66(10)$ & $73(8)^{*}$ & $<0,001$ \\
Sexo & $147: 73$ & $57: 33$ & $<0,001$ \\
FEV1 $(\%$ predicted $)$ & $46,3(18,6)$ & $40,5(19,6)$ & 0,013 \\
SMM $(\mathrm{kg})$ & $26,6(6,9)$ & $18,9(5,1)^{*}$ & $<0,001$ \\
SMI $\left(\mathrm{kg} / \mathrm{m}^{2}\right)$ & $9,2(1,8)$ & $6,9(1,3)^{*}$ & $<0,001$ \\
Handgrip $(\mathrm{kg})$ & $33,9(8,6)$ & $21,5(7,3)^{*}$ & $<0,001$ \\
4MGS $(\mathrm{m} / \mathrm{s})$ & $1,07(0,16)$ & $0,77(0,22)^{*}$ & $<0,001$ \\
Actividad física objetiva $(\mathrm{n}=129)$ & & & $<0,001$ \\
Conteo de pasos diarios & $5.127(2.475 ; 6,815)$ & $1.482(1.205,3.301)$ & \\
\hline
\end{tabular}

SMI= índice muscular esquelético; SMM= masa muscular esquelética; 4MGS= velocidad de la marcha en 4 metros.

significativamente los siguientes parámetros: índice muscular esquelético (SMI), fuerza de prensión y 4 MGS (velocidad de la marcha) (con promedios de mejoría de $0,11 \mathrm{~kg} / \mathrm{m}^{2}, 2,08 \mathrm{~kg}$ y $0,12 \mathrm{~m} / \mathrm{s}$, respectivamente) y en consecuencia 12 de 43 pacientes (correspondientes al 28\%) ya no cumplieron con los criterios del EWGSOP para sarcopenia (tabla 2).

\section{Conclusión}

En EPOC se reportó una prevalencia alta de sarcopenia, siendo esta directamente proporcional a la edad y a la severidad. Los pacientes con sarcopenia tienen peor estado físico y peor estado funcional.
Los pacientes con EPOC y sarcopenia respondieron bien a la rehabilitación pulmonar, con mejoras en el rendimiento funcional, la capacidad de ejercicio, la fuerza de las extremidades superiores y el estado de salud, similares a los obtenidos por los pacientes sin sarcopenia.

De esta manera, este estudio demostró que la rehabilitación pulmonar puede revertir la sarcopenia en pacientes seleccionados, en particular, aquellos con índice de masa esquelética o el rendimiento funcional está cerca del umbral de corte al inicio del estudio.

Tabla 2. Comparación de la respuesta a la rehabilitación pulmonar en sujetos con y sin sarcopenia en una muestra total de pacientes con enfermedad pulmonar obstructiva crónica.

\begin{tabular}{|c|c|c|c|c|c|c|}
\hline \multicolumn{4}{|c|}{ Línea de base } & \multicolumn{3}{|c|}{ Cambio con rehabilitación pulmonar } \\
\hline & Sin sarcopenia & Sarcopenia & Valor de $p$ & Sin sarcopenia & Sarcopenia & Valor de $p$ \\
\hline $\mathrm{SMI}\left(\mathrm{kg} / \mathrm{m}^{2}\right)$ & $8,8(8,3$ a 9,3$)$ & $7,0(6,6$ a7,3) & $<0,001$ & $0,0(-0,1$ a 0,2$)$ & $0,1(0,0$ a 0,2$)$ & 0,431 \\
\hline Handgrip (kg) & $25,7(22,8$ a 28,4$)$ & $\begin{array}{r}21,2(19,2 \text { a } \\
23,3)\end{array}$ & 0,021 & $1,6(0,5$ a 2,6$)$ & $2,1(1,3$ a 2,9$)$ & 0,516 \\
\hline 4MGS $(\mathrm{m} / \mathrm{s})$ & $0,86(0,80$ a 0,91$)$ & $\begin{array}{r}0,83(0,77 \mathrm{a} \\
0,89)\end{array}$ & 0,511 & $\begin{array}{r}0,11(0,07 \text { a } \\
0,14)\end{array}$ & $\begin{array}{r}0,12(0,08 a \\
0,16)\end{array}$ & 0,552 \\
\hline
\end{tabular}

SMI= índice muscular esquelético; 4MGS= velocidad de la marcha en 4 metros. 


\section{Comentario}

Desde hace tiempo el manejo de las enfermedades respiratorias crónicas se ha enfocado en el manejo de los síntomas con medidas farmacológicas dirigidas a los cambios fisiopatológicos del pulmón, pero ahora entendemos que estas más que localizadas son sistémicas y complejas, y que además requieren intervenciones no farmacológicas como la rehabilitación pulmonar que permitan mejorar la capacidad de las personas de desempeñarse en su vida diaria, ser independientes y seguir activos en contexto social y laboral para preservar el bienestar y la calidad de vida $(1,2)$.

El objetivo de los programas de rehabilitación pulmonar es revertir algunas manifestaciones extrapulmonares comunes de la EPOC, entre ellas la intolerancia al ejercicio, el mal estado nutricional y la mala autopercepción de calidad de vida. En la actualidad este tipo de terapia no farmacológica es considerada uno de los componentes principales del manejo de esta enfermedad (3-5).

La pérdida de la masa muscular se observa en una gran proporción de los pacientes con EPOC, pero no siempre se acompaña de pérdida de peso y podemos referirnos a este fenómeno como el fenotipo sarcopénico. Múltiples estudios muestran que las personas con baja masa muscular tienen peor percepción de calidad de vida y peor desempeño físico, y que ello es un factor pronóstico de malos desenlaces $(6,7)$.

Es claro que los pacientes con EPOC tienen limitación para la actividad física, pero ahora hay evidencia que avala de forma contundente que la disfunción en el músculo esquelético contribuye también a esta intolerancia (8-11). Se ha visto una reducción en el tamaño de los cuádriceps en la mayoría de los pacientes con EPOC y que esto se asocia a alteración en la marcha, dificultad para desplazarse y mayor consumo de oxígeno (2). La fisiopatología de la disfunción del músculo esquelético en la EPOC es compleja y hasta el momento no se entiende por completo. En los pacientes con EPOC se han descrito varias alteraciones estructurales, incluida la masa muscular disminuida y el cambio de las fibras tipo I a fibras tipo II, específicamente en los vastos laterales (12). Además, las actividades reducidas de enzimas oxidativas (citrato sintasa, succinato deshidrogenasa y 3-hidroxiacil-CoA deshidrogenasa) se detectaron sistemáticamente en las biopsias musculares del cuádriceps de pacientes con EPOC moderada a grave en comparación con los controles (13).

En Colombia ya existen programas de rehabilitación pulmonar, pero aunque funcionan de forma exitosa, son muy reducidos y no están disponibles en todo el territorio nacional ni para toda la población que los requiere. El principal problema actual es el desconocimiento por parte de muchos médicos y entidades de salud acerca de las ventajas en el cuidado de los pacientes y el ahorro de recursos que estas intervenciones generan, lo que hace necesario que haya un mayor estudio, interés y conocimiento para su implementación efectiva y eficaz.

Como medidas prácticas para la consulta del neumólogo, es posible recomendar la aplicación de al menos dos medidas de tamizaje para la búsqueda clínica de sarcopenia; medir el perímetro de pantorrilla $10 \mathrm{~cm}$ por debajo de la patela de la pierna dominante. Si el valor es menor a $31 \mathrm{~cm}$ se considera que es baja la masa muscular. También la velocidad de la marcha de $4 \mathrm{~m}$ o $6 \mathrm{~m}$ puede ser muy útil y es considerada la medida de ejecución física más importante. Para una medición de $4 \mathrm{~m}$ se debe de disponer de 6 metros de espacio plano y recto. El primer y último metro son espacios de impulso y frenado, por lo que no se incluyen en la medición. Solo se medirá la velocidad pura y se expresará en metros/segundo $(\mathrm{m} / \mathrm{s})$. Se considera a nivel internacional que un valor menor a $0,8 \mathrm{~m} / \mathrm{s}$ es anormal y se relaciona con sarcopenia, la cual es un factor de riesgo para caídas, pérdida funcional a largo plazo e igualmente es la puerta de entrada para un estado de fragilidad.

Las mediciones anteriores identificarán el paciente con alta probabilidad de tener sarcopenia y requerirá de una evaluación integral que incluya búsqueda de causas de pérdida de masa muscular, evaluación nutricional e incluso análisis de recursos sociales. $\mathrm{Si}$ se trata de un anciano, se recomienda evaluación por Geriatría.

El objetivo principal de la intervención en rehabilitación es evitar un mayor deterioro funcional o 
revertirlo parcial o totalmente. La prescripción del ejercicio requiere de una juiciosa evaluación de múltiples sistemas (cardiaco, pulmonar, osteoarticular), evitando generar un mayor daño o una experiencia desagradable por dolor. En todos los casos se debe dar prioridad al manejo no farmacológico en sarcopenia (nutrición adecuada y ejercicio de fuerza).

\section{Conflictos de intereses}

Los autores declaran no tener conflictos de interés.

\section{Bibliografía}

1. Cruz-Jentoft AJ, Baeyens JP, Bauer JM, Boirie Y, Cederholm T, Landi F, et al. Sarcopenia: European consensus on definition and diagnosis: Report of the European Working Group on Sarcopenia in Older People. Age and Ageing. 2010;39:412-23.

2. Jones SE, Maddocks M, Kon SS, Canavan JL, Nolan CM, Clark AL, et al. Sarcopenia in COPD: prevalence, clinical correlates and response to pulmonary rehabilitation. Thorax. 2015;70:213-8.

3. Goldstein G, Shelly C. Does the right hemisphere age more rapidly than the left? J Clin Neuropsychol. 1981;3:65-78.

4. Schols AM, Soeters PB, Mostert R, Pluymers RJ, Wouters EF. Physiologic effects of nutritional support and anabolic steroids in patients with chronic obstructive pulmonary disease. A placebo-controlled randomized trial. Am J Resp Crit Care Med. 1995;152:1268-74.

5. Lacasse Y, Brosseau L, Milne S, Martin S, Wong E, Guyatt GH, et al Pulmonary rehabilitation for chronic obstructive pulmonary disease. The Cochrane database of systematic reviews. 2002:Cd003793.

6. Baarends EM, Schols AM, Mostert R, Wouters EF. Peak exercise response in relation to tissue depletion in patients with chronic obstructive pulmonary disease. Eur Respir J. 1997;10:2807-13.

7. Mostert R, Goris A, Weling-Scheepers C, Wouters EF, Schols AM. Tissue depletion and health related quality of life in patients with chronic obstructive pulmonary disease. Respiratory Med. 2000;94:859-67.

8. Samper-Ternet R, Reyes-Ortiz C, Ottenbacherc K, Cano C. Frailty and Sarcopenia in Colombia: Results from the SABE Bogotá Study 2014.

9. Marcell TJ. Sarcopenia: causes, consequences, and preventions. JGerontolA BiolSciMedSci. 2003;58:M911-M6.

10. Rosenberg IH. Sarcopenia: origins and clinical relevance. Clin Geriatr Med. 2011;27:337-9.

11. Rolland Y, Czerwinski S, Abellan VK, Morley JE, Cesari M, Onder G, et al. Sarcopenia: its assessment, etiology, pathogenesis, consequences and future perspectives. J NutrHealth Aging. 2008;12:433-50.

12. Gosker HR, Zeegers MP, Wouters EF, Schols AM. Muscle fibre type shifting in the vastus lateralis of patients with COPD is associated with disease severity: a systematic review and meta-analysis. Thorax. 2007;62:944-9.

13. Jakobsson P, Jorfeldt L, Henriksson J. Metabolic enzyme activity in the quadriceps femoris muscle in patients with severe chronic obstructive pulmonary disease. Am J Resp Crit Care Med. 1995;151:374-7. 